2019-01-01

\title{
An improved prediction of the effective range of stress intensity factor in fatigue crack growth
}

\author{
Yang, B
}

http://hdl.handle.net/10026.1/15914

10.1007/978-3-030-13980-3_17

All content in PEARL is protected by copyright law. Author manuscripts are made available in accordance with publisher policies. Please cite only the published version using the details provided on the item record or document. In the absence of an open licence (e.g. Creative Commons), permissions for further reuse of content should be sought from the publisher or author. 


\title{
An Improved Prediction of the Effective Range of Stress Intensity Factor in Fatigue Crack Growth
}

\author{
Bing Yang ${ }^{\mathrm{a}, \dagger}$, M.N. James ${ }^{\mathrm{b}, \mathrm{c}^{*}}$, Yongfang Huang ${ }^{\mathrm{d}, \dagger}$, J.M. Vasco-Olmo ${ }^{\mathrm{e}}$ and F.A. \\ Díaz ${ }^{\mathrm{e}}$ \\ ${ }^{a}$ State Key Laboratory of Traction Power, Southwest Jiaotong University, Chengdu, China \\ ${ }^{\mathrm{b}}$ School of Engineering, University of Plymouth, Plymouth, England \\ ${ }^{c}$ Department of Mechanical Engineering, Nelson Mandela Metropolitan University, Port Eliza- \\ beth, South Africa \\ ${ }^{\mathrm{d}}$ Department of Aeronautics, Xiamen University, Xiamen, China \\ e Departamento de Ingeniería Mecánica y Minera, University of Jaén, Jaén, Spain \\ mjames@plymouth.ac.uk
}

\begin{abstract}
This paper will summarise the results obtained to date and which demonstrate that the mesoscale CJP model of crack tip fields is capable of providing an improved correlation of fatigue crack growth rates across a range of stress ratios and specimen geometries, compared with the standard stress intensity factor calculations.
\end{abstract}

Keywords: crack tip field model, digital image correlation, shielding.

\section{Introduction}

The CJP model is a meso-scale model of crack tip displacement and stress that was proposed a few years ago as an attempt to better characterise the elastic forces induced by the plastic enclave that surrounds a growing fatigue crack and hence enable direct prediction of the effective range of crack driving force. The model was a development of earlier work that had achieved some success in measuring the wake contact pressure arising from the plastic enclave that surrounds a growing fatigue crack [1]. The theoretical model in Mode I loading was extended from a stress-based version that could be fitted to full-field photoelastic images of the crack tip region [2, 3], to one that utilised digital image correlation and could be applied directly to displacement fields on metallic specimens [4]. The next step in the development of the model extended it to deal with combined Mode I and Mode II loading which, in principle, would open up its use to include characterising surface roughness-induced shielding as well as plasticity-induced shielding [5].

Experimental verification of the concepts in the CJP model followed a little more slowly than the theoretical developments, reflecting factors such the complexity of phase-stepping photoelastic fatigue experiments, the development of software necessary to automate the CJP model solution from photoelastic and DIC images, and the training of $\mathrm{PhD}$ students. Over the last several years, however, researchers from the 
University of Jaen in Spain, Gifu University in Japan, Southwest Jiaotong University and Xiamen University in China have been making considerable progress in experimental verification of the model $[6,7]$

This paper will summarise the results obtained to date and which demonstrate that the CJP model appears capable of providing an improved correlation of fatigue crack growth rates across a range of stress ratios and specimen geometries, compared with the standard stress intensity factor calculations. The model also appears to correctly characterise both plastic zone shape and size and this paper will also briefly discuss the data obtained from overload experiments.

\section{Background to the Model}

The objective of the work described in reference [2] was to identify the real influence or effect on the applied elastic field, of stresses arising from plastic deformation associated with crack growth. Reference [4] notes that the CJP model essentially treats the crack as a notional plastic inclusion in an elastic body. This approach leads to the definition of a stress intensity factor perpendicular to the crack plane, called $K_{F}$, which drives crack growth in an analogous fashion to $K_{I}$ and is modified by the incorporation of shielding force components acting perpendicular to the crack. The shielding effect of the plastic enclave is considered via a new retarding stress intensity factor $K_{R}$ that is defined to account for forces in the plane of the crack that act to retard the crack, and the model also defines an interfacial shear stress intensity factor, $K_{S}$, which is included to capture compatibility-induced shear components of shielding that would perhaps be more applicable to Mode II or III loading. This paper will focus on $K_{F}$ and $K_{R}$ that have been shown to be applicable to Mode I fatigue crack growth. The model also defines a value for the $T$-stress as this parameter has certain characteristics that affect fatigue crack growth rate; as stated in reference [8] these depend on the sign and magnitude of the $T$-stress and can include substantially changing the size and shape of the plane strain crack tip plastic zone, a decrease in fatigue crack growth rate in the Paris law regime with increasing $T$-stress (this reflects the fact that higher positive values of $T$-stress imply higher constraint and smaller plastic zone size), and crack path influences (negative $T$-stress values can stabilise the crack path while, in contrast, positive $T$-stress values induce crack bifurcation). Reference 4 discusses the $T$-stress in the context of using the CJP model to characterise fatigue crack growth in polycarbonate CT specimens. The model can also be used to investigate the effect of changing values of $T$ stress on the crack tip field as illustrated in reference [2] for the case of photoelastic fringes showing difference in principal stress. Clearly, for a crack tip model to more accurately define plastic zone size and shape, the $T$-stress is an important parameter.

The intention in developing the CJP model was to elucidate the role and influence of factors such as compatibility-induced interfacial shear stresses and wake contact forces on the effective range of stress intensity factor and any resulting changes observed in the fatigue crack growth rate. There was therefore a dual aim of improved mechanistic understanding of fatigue phenomena such as crack closure, plasticity-in- 
duced shielding and overload effects, together with obtaining an improved characterisation of fatigue crack growth in situations where these phenomena are occurring. As an example of improved understanding of the mechanisms that underlie observed phenomena, reference [2] indicates that the introduction of interfacial shear stresses causes migration along the crack path of the join between the photoelastic fringe loops on either side of the crack path. This is perhaps more pronounced in the crack wake, but both ahead and behind the crack, the shape of the join between the loops of common fringe order changes from a sharp ' $\mathrm{V}$ ' to more of a ' $\mathrm{U}$ ' shape. This phenomenon has been observed before in photoelastic images in the presence of crack closure but the discussion in reference [2] is probably the first time that it could be assigned, fairly reasonably, to the effect of interfacial shear stresses. Thus the model offers significant potential in terms of obtaining a better physical understanding of the potential role of the various forces in plasticity-induced shielding.

Further independent evidence of the utility of the model in providing predictions of the effective range of stress intensity factor comes from work recently published by Nowell et al [9]. In reference 9 they discuss the CJP model and present a slightly simplified version of the force diagram given in reference 2 . They go on to demonstrate, using DIC data obtained from a growing fatigue crack, that the combined $K_{F}+$ $K_{R}$ parameter predicts, a priori, very similar values of $\Delta K_{\text {eff }}$ throughout a fatigue cycle to those measured experimentally on an existing crack with their technique. Nowell et al [9] express reservations regarding the split of $\Delta K$ into the $K_{F}$ and $K_{R}$ terms although they note in their paper that the split into what they call "applied and residual terms" may be helpful, even for measurements taken very close to the crack tip.

In terms of providing an improved characterisation of fatigue crack growth rate, sufficient experimental work has now been performed across a range of stress ratio values, specimen geometries and materials to verify that the model does indeed achieve this, compared with data obtained using the standard Irwin stress intensity value.

This paper will demonstrate this by presenting experimental fatigue crack growth rate data obtained at Plymouth and Jaen over the last two years and it will also summarise current work aimed at predicting fatigue crack growth rates using the CJP model and a calibration curve approach. Other ongoing work has considered the capability of the CJP model to characterise plastic zone size and shape through the application of overload cycles during constant amplitude (CA) fatigue [10], and compared the size and shape results with those given by other commonly used elastic models of crack tip stresses [11]. The results demonstrate that the CJP model provides a very good characterisation of plastic zone size and shape during CA loading and throughout an overload event, while the accurate prediction of effective stress intensity factor range and the insights offered into the mechanisms operating during shielding allow the influence of other potential causes of crack growth changes during an overload to be identified [10]. 


\section{Specimen Geometries and Experimental Techniques}

Recent work has focussed on Grade 2 (commercially pure - CP) titanium with a measured yield strength of $390 \mathrm{MPa}$ and a tensile strength of $448 \mathrm{MPa}$. The elastic Young's modulus $\mathrm{E}=105 \mathrm{GPa}$ and Poisson's ratio $v=0.33$. Fig. 1 shows the three specimen geometries that were used in the tests, i.e. compact tension (CT), double edge-notched tension (DENT) and centre-cracked tension (CCT). Fatigue testing was performed on an ElectroPuls E3000 with the relevant details given in Table 1.

The CCD camera used to acquire DIC images was equipped with a 10x magnification macro-zoom lens to provide the necessary spatial resolution in the measurement region surrounding the crack tip. The field of view was 1624 pixels by 1202 pixels (approximately $13.68 \mathrm{~mm}$ by $10.12 \mathrm{~mm}$, with slight specimen-to-specimen variations), giving the spatial resolution for each specimen shown in Table 1. Data acquisition involved periodically pausing the fatigue cycling and applying stepwise loading through a fatigue cycle, making DIC measurements at each step and crack tip positon was recorded using a travelling microscope on the reverse side of the specimen (resolution $\approx 10 \mu \mathrm{m}$ ). Full experimental and analysis details are given in reference 7 .

Fig. 2 a presents the crack growth rate data characterised in terms of $\Delta K$, while Fig. $2 \mathrm{~b}$ presents the same information characterised using $\Delta K_{C J P}$, which is defined as:

$$
\Delta K_{C J P}=\left(K_{F, \max }-K_{R, \max }\right)-\left(K_{F, \min }-K_{R, \min }\right)
$$

The level of plasticity-induced crack tip shielding in CP titanium is fairly low and hence, on a log-log plot the improvement in the characterisation into a single curve may not appear very high, reflecting the low level of plasticity-induced shielding in CP titanium. However, statistical analysis demonstrates a significant improvement in terms of fitting the data with a single straight line, e.g. from a regression parameter of 0.9504 for the DENT, CT and CCT curves using $\Delta K$ to 0.9861 using $\Delta K_{C J P}$. It is also the case that the CJP crack tip field model does not require the incorporation of compliancebased geometry-correction factors in the calculation of stress intensity. Reference 7 has shown that there are simple relationships between $\Delta K_{C J P}$ and $\Delta K$ using calibration curves that relate the values of the parameters in the CJP model to the standard $\Delta K$ value. These relationships have been determined for CT and DENT specimens of the $\mathrm{CP}$ titanium alloy, and they would be affected by influences that change the stress component parameters A, B or D, i.e. changes in the forces that contribute to shielding.

\section{Concluding Remarks}

The work described in reference 7 and the additional data on CCT specimens presented here give confidence that the CJP model has significant potential to improve crack growth characterisation where plasticity-induced shielding of the crack tip is occurring. The work reported in reference 10 has shown that the CJP model can be used to obtain accurate predictions of both the effective range of stress intensity factor and 
the changes in plastic zone size and shape that occur during variable amplitude fatigue, and to then assess how well the changes in crack growth rate can be correlated with the effects of plasticity-induced closure.

\section{References}

1. Pacey, M.N., Patterson, E.A. and James, M.N.: A new photoelastic model for studying fatigue crack closure, Experimental Mechanics 45(1), 42-52 (2005).

2. Christopher, C.J., James, M.N., Patterson, E.A. and Tee, K.F.: Towards a new model of crack tip stress fields, International Journal of Fracture 148, 361-371 (2007).

3. Christopher, C.J., James, M.N., Patterson, E.A. and Tee, K.F.: A quantitative evaluation of fatigue crack shielding forces using photoelasticity, Engineering Fracture Mechanics 75, 4190-4199 (2008).

4. James, M.N., Christopher, C.J., Yanwei Lu and Patterson, E.A.: Local Crack Plasticity and its Influences on the Global Elastic Stress Field, International Journal of Fatigue 46, 4-15 (2013)

5. Christopher, C.J., Laboviciute, G., James, M.N. and Patterson, E.A.: Extension of the CJP model to mixed Mode I and Mode II, Frattura ed Integrità Strutturale 7 25, 161-166 (2013).

6. James, M.N., Christopher, C.J., Díaz Garrido, F.A., Vasco-Olmo, J.M., Toshifumi Kakiuchi and Patterson, E.A.: Interpretation of plasticity effects using the CJP crack tip field model, Solid State Phenomena, 258, 117-124 (2016) Proceedings of the 8th International Conference on Materials Structure \& Micromechanics of Fracture, Brno, Czech Republic, 27-29 June 2016.

7. Yang, Bing, Vasco-Olmo, J.M., Díaz, F.A. and James, M.N.: A more effective rationalisation of fatigue crack growth rate data for various specimen geometries and stress ratios using the CJP model, submitted to the International Journal of Fatigue (2018).

8. Hadj Meliani M, Azari Z, Pluvinage G, Matvienko YG.: The effective T-stress estimation and crack paths emanating from U-notches, Engineering Fracture Mechanics 77, 1682-1692 (2010).

9. Nowell1, D., Dragnevski, K.I. and O'Connor, S.J.: Investigation of Fatigue Crack Models by Micro-scale Measurement of Crack Tip Deformation, accepted by the International Journal of Fatigue, (2018).

10. Vasco-Olmo, J.M., Díaz, F.A., James, M.N. and Bing Yang: Crack tip plastic zone evolution during an overload cycle and the contribution of plasticity-induced shielding to crack growth rate changes, in press: Fatigue \& Fracture of Engineering Materials \& Structures, (2018).

11. Vasco-Olmo, J.M., James, M.N., Christopher, C.J., Patterson, E.A. and Díaz, F.A.: Assessment of crack tip plastic zone size and shape and its influence on crack tip shielding, Fatigue \& Fracture of Engineering Materials \& Structures, 39(8), 969-981 (2016).

Table 1. Fatigue test parameters used in this work

\begin{tabular}{cccc}
\hline Specimen & $P_{\max }(\mathrm{N})$ & $R$ & DIC Spatial Resolution $(\mu \mathrm{m} / \mathrm{pixel})$ \\
\hline $\mathrm{CT} 1$ & 700 & 0.1 & 8.33 \\
$\mathrm{CT} 2$ & 700 & 0.3 & 8.33 \\
$\mathrm{CT} 3$ & 700 & 0.6 & 8.22
\end{tabular}




\begin{tabular}{cccc} 
DENT1 & 2200 & 0.05 & 8.62 \\
DENT2 & 2200 & 0.3 & 8.61 \\
CCT1 & 2200 & 0.1 & 8.88 \\
CCT2 & 2200 & 0.3 & 7.36 \\
CCT3 & 2200 & 0.6 & 7.65 \\
\hline
\end{tabular}

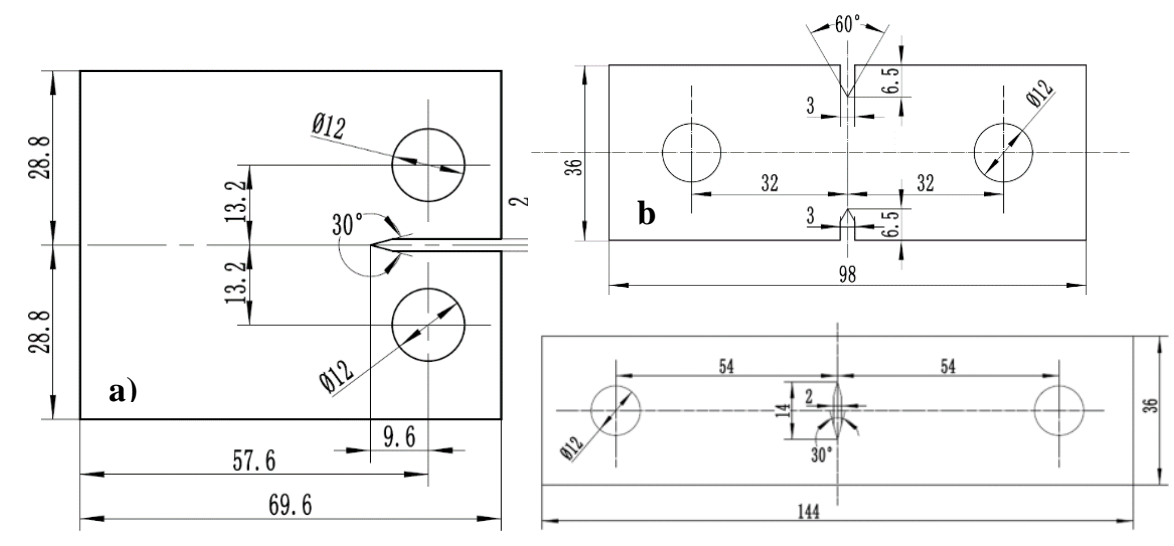

Fig.1. The three specimen geometries used in this work: a) CT; b) DENT; c) CCT.
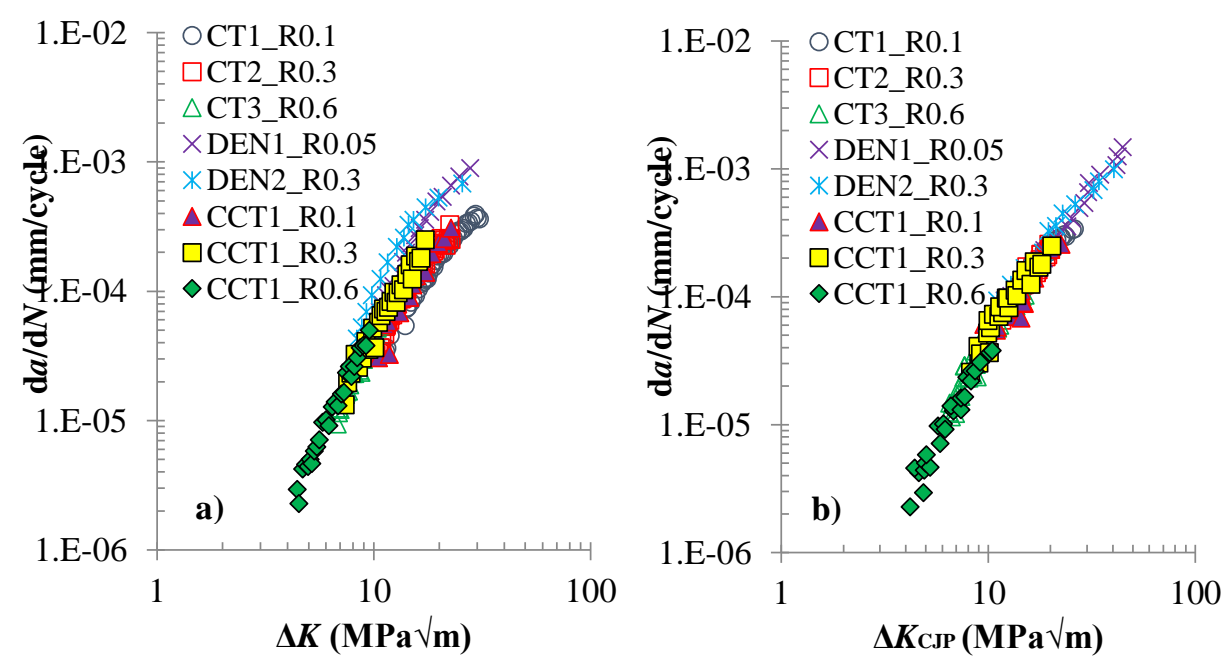

Fig. 2. Fatigue crack growth rate data characterised by a) the standard definition of $\Delta K$; b) $\Delta K_{C J P}$. 